Interdisciplinary Risk Research: Remote Sensing, Radar Altimetry, GIS, etc

\title{
Integration of the Electrical Resistivity Tomography and Seismic Refraction Survey Data to Identify the Structure of Oxidized Waterlogged Zones of the Mine Tailings (Western Siberia, Russia)
}

\author{
Sofiya Grakhova, Petr Dergach, Nikolay Yurkevich, Dmitry Kucher \\ IPGG SB RAS, 630090, Novosibirsk, Russia
}

doi: https://doi.org/10.21467/abstracts.93.69

\begin{abstract}
Introduction. The current situation of mine wastes, ore dressing tailings, and slag from the metallurgical industry in direct access to oxidizing and transporting factors has radically changed the environmental conditions in mining regions, not only in Russia but also throughout the world. Water transport of potentially toxic elements from dumps is gaining momentum over time due to the destruction of the tailings mineral matrix and the transition of elements to mobile forms. The formation of highly mineralized solutions inside the body of dumps and the direction of their subsequent migration in the external environment are issues without which it is impossible to develop protective measures against the harmful effects of acidic drainage water [1]. To assess damage and monitor such objects, it is necessary to develop methods for continuous monitoring and testing. For several years we carried out electrotomography and geochemical studies on the Ur dump, and in 2019 the seismic tomography technique was tested.
\end{abstract}

Study object located in the Barit village, Kemerovo region, it was formed in the 1930s. (Fig. 1). It is composed of barite-pyrite granularity from the oxidized zone of the Novo-Ursk deposit pyrite after gold extraction by cyanidation. The total iron content (in terms of $\mathrm{Fe}_{2} \mathrm{O}_{3}$ ) is $15 \%$, sulfur (in terms of $\mathrm{SO}_{3}$ ) is $10 \%$. A natural stream flows near its foot, becoming after a dump an acidic sulphate highly mineralizedsolutionwith $\mathrm{pH}$ 2.1, specific conductivity $3.9 \mathrm{mS} / \mathrm{cm}$ and high concentrations of metals and metalloids [2].

Methods. The electrical resistivity tomography (ERT) and Seismic refraction survey were carried out at the Ur dump in 2019.

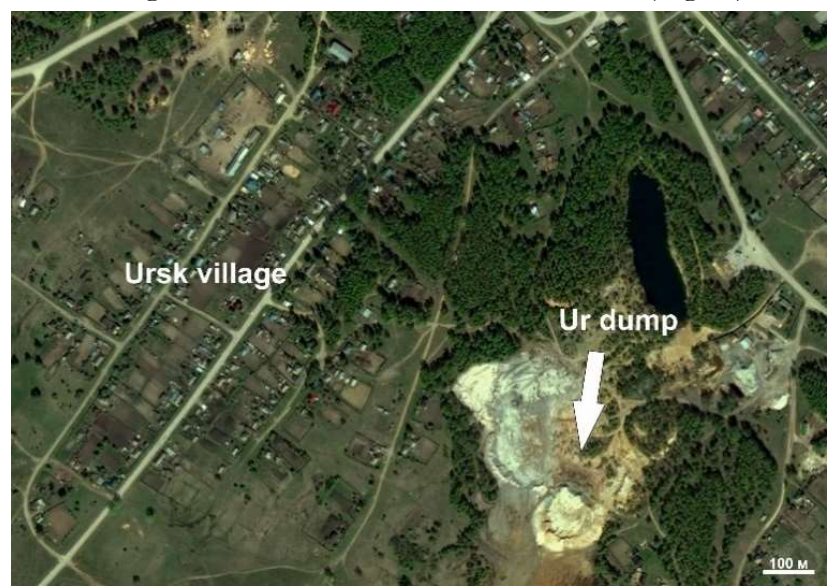

Figure 1. The layout of the Ur dump relative to the Ursk village.

ERT was carried out by the multi-electrode electrical resistivity and induced polarization imaging instruments «SibER-48»(LLC«KB Electrometry», Novosibirsk) in cloudless weather with a slight wind. A single braid was used in the profile, the step between the electrodes was $5 \mathrm{~m}$, the total length of the profile was $120 \mathrm{~m}$, so the most informative depth is $5-10 \mathrm{~m}$.

Seismic observations were carried out along a 180-meter linear section with a distance of $1 \mathrm{~m}$ between adjacent geophones. Excitation was carried out using a sledgehammer on a metal plate with a step of $5 \mathrm{~m}$.

C) 2020 Copyright held by the author(s). Published by AIJR Publisher in "Abstracts of The Second Eurasian RISK-2020 Conference and Symposium" April 12- 19, 2020, Tbilisi, Georgia. Jointly organized by AMIR Technical Services LLC, Georgian Technical University, Institute of Geography (Kazakhstan) and Russian Institute of Petroleum Geology and Geophysics. 
The Second Eurasian RISK-2020 Conference and Symposium

Seismic data processing was carried out by two methods: seismo-tomography [3] and Generalized reciprocal method GRM [4].As a result, a smooth velocity model was obtained (Fig. 2), and the depths to the boundaries between layers with different acoustic stiffness were determined (marked by red lines).

Results. The center of the ERT cross-section was at the transition of the dump slope to a flat part (Fig. 2). Integration of electro- and seismo - tomography methods showed an amazing convergence of results. The bedrocks occur at shallow depths. At the same time, the dump itself is the most conductive, and therefore highly mineralized, watered environment. Obviously, melting snow as well as rainfall wash out the dump, creating a threat to groundwater. In addition, it was possible to identify the boundaries corresponding to the surface friable layer, the base of the dump, and also the top of bed rocks according to seismic data. This information can be used to invert ET data. In the future, it is planned to conduct seismic investigations along the same cross-section using share waves. It will allow identify longitudinal and shear wave velocities ratio $V_{s} / V_{p}$, which is an indicator of the water saturation of rocks.

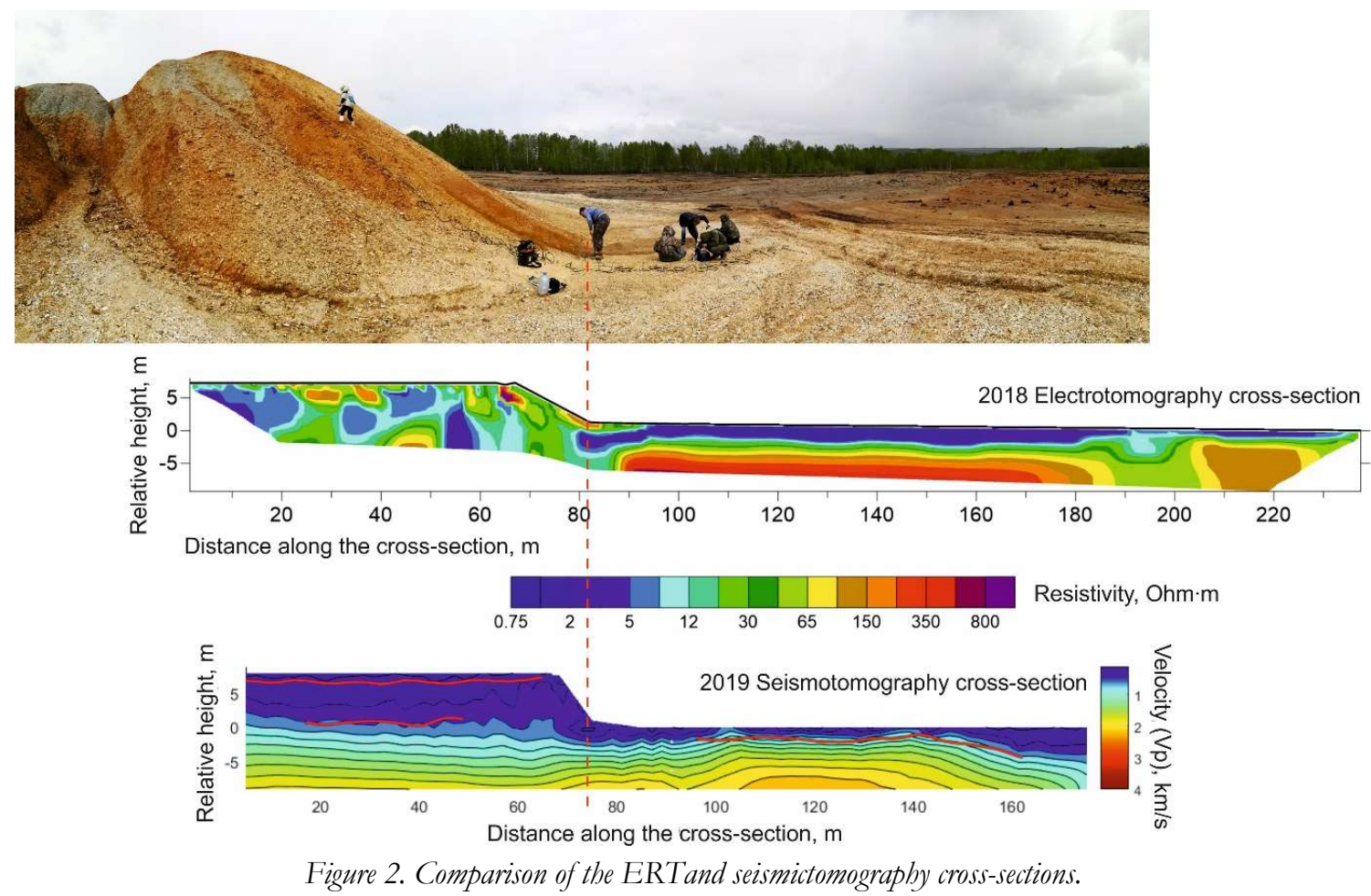

Funding. This research was funded by the RUSSIAN FOUNDATION FOR BASIC RESEARCH, grant number 20-05-00336 and a BASIC RESEARCH PROJECT, project number 0266-2019-0008.

\section{References}

[1] Olenchenko V.V., Kucher D.O., Bortnikova S.B., Edelev A.V., Gas'kova O.L., Gora M.P. Vertical and lateral spreading of highly mineralized acid drainage solutions (Ur dump, Salair): Electrical resistivity tomography and hydrogeochemical data // Russian Geology and Geophysics. 2016. T. 57. № 4. C. 617-628.

[2] Yurkevich N.V., Olenchenko V.V., Bortnikova S.B., Edelev A.V., Saeva O.P. The biotic processes in sulfide bearing mine tailings in winter according to geochemical and geophysical data // Interexpo Geo-Siberia, 4. 2018.

[3] Koulakov I., Kopp H. Combined tomographic forward and inverse modeling of active seismic refraction profiling data // AGU Fall Meeting Abstracts. - 2008.

[4] Palmer D. An introduction to the generalized reciprocal method of seismic refraction interpretation //Geophysics. -1981. - T. 46. - №. 11. - C. 1508-1518. 